\title{
Gold and Silver-Catalyzed Highly Regioselective Addition of Active Methylenes to Dienes, Triene, and Cyclic Enol Ethers
}

\author{
Rene-Viet Nguyen, Xiao-Quan Yao, D. Scott Bohle, ${ }^{\dagger}$ and Chao-Jun Li* \\ Department of Chemistry, McGill University, 801 Sherbrooke St. West, Montreal, QC H3A \\ 2K6 Canada; cj.li@mcgill.ca
}

\section{Supporting information}

All experiments were carried out under a nitrogen atmosphere. Dichloromethane was dried using $\mathrm{CaH}_{2}$. Column chromatography was performed over Sorbent silica gel $30-60 \mu \mathrm{m} .{ }^{1} \mathrm{H}-$ NMR spectra were acquired by Varian 400 and $100 \mathrm{MHz}$ or 300 and $75 \mathrm{MHz}$, respectively, and referenced to the internal solvent signals. IR spectra were recorded by ABB Bomem MB100 spectrometer. Mass spectra data and HRMS were obtained by Kratos MS25RFA Mass Spectometer. X-ray determination was resolved by Rigaku AFC63 four-circle diffractometer.

\section{Typical experimental procedure(entry 1, table 2):}

A solution of $\mathrm{AuCl}_{3}(7.6 \mathrm{mg}, 0.025 \mathrm{mmol})$ and $\mathrm{AgOTf}(19.3 \mathrm{mg}, 0.075 \mathrm{mmol})$ was stirred in dichloromethane $(2 \mathrm{~mL})$ at room temperature for $2 \mathrm{hrs}$. Dibenzoylmethane $(112 \mathrm{mg}, 0.5 \mathrm{mmol})$ was then added which was followed by the addition of cyclooctadiene $(128 \mu \mathrm{L}, 1 \mathrm{mmol})$ via a syringe pump over $5 \mathrm{~h}$ and the resulting solution was stirred overnight. The solvent was removed under reduced pressure and the product was isolated via column chromatography on silica gel(gradiant eluent: hexane: ethyl acetate $=60: 1$ to $2: 1$ ). 
2-Cyclooct-2-enyl-1,3-diphenyl-propane-1,3-dione.

$\mathrm{IR}(\mathrm{KBr}$

plate): $v_{\max } 2929,2851,1688,1594,1447,1239,976,776,715 \mathrm{~cm}^{-}$

${ }^{1}$; ${ }^{1} \mathrm{H} \mathrm{NMR}\left(\mathrm{CDCl}_{3}, 300 \mathrm{MHz}, \mathrm{ppm}\right): \delta=8.04-7.96(\mathrm{~m}, 5 \mathrm{H}), 7.46-$

7.39(m, 5H), 5.63(m, 1H) 5.31-5.22(m, 2H), 3.83(m, 1H), 2.30(m,

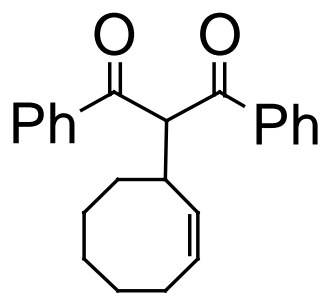

$1 \mathrm{H}), 2.04(\mathrm{~m}, 1 \mathrm{H}), 1.87-1.22(\mathrm{~m}, 10 \mathrm{H}) ;{ }^{13} \mathrm{C} \mathrm{NMR}\left(\mathrm{CDCl}_{3}, 75 \mathrm{MHz}, \mathrm{ppm}\right): \delta=195.5,137.3$, 133.5, 130.5, 129.0, 63.7, 38.2, 34.5, 31.3, 29.9, 27.1, 25.8; MS(EI): $\mathrm{m} / \mathrm{z}(\%) 332\left(\mathrm{M}^{+}\right)$, 227(100), 105, 77; HRMS calc'd for $\mathrm{C}_{23} \mathrm{H}_{24} \mathrm{O}_{2}$ : 332.1776; found, 332.1771.

2-Cyclohepta-2,4-dienyl-1,3-diphenyl-propane-1,3-dione. $\quad \operatorname{IR}(\mathrm{KBr}$ plate): $\mathrm{v}_{\max }=3008,2915,2352,1696,1647,1595,1442,1269,991,758$ $\mathrm{cm}^{-1} ;{ }^{1} \mathrm{H} \mathrm{NMR}\left(\mathrm{CDCl}_{3}, 300 \mathrm{MHz}, \mathrm{ppm}\right): \delta=7.98-7.39(\mathrm{~m}, 4 \mathrm{H}), 7.55-$ 7.40(m, 6H), 5.90(m, 2H), 5.67(m, 2H), 5.54(d, 1H, J=9.9 Hz), 3.74(m,<smiles>O=C(C(=O)C1C=CC=CCC1)c1ccccc1</smiles>
$1 \mathrm{H}), 2.42(\mathrm{~m}, 2 \mathrm{H}), 1.95(\mathrm{~m}, 2 \mathrm{H}) ;{ }^{13} \mathrm{C} \mathrm{NMR}\left(\mathrm{CDCl}_{3}, 100 \mathrm{MHz}, \mathrm{ppm}\right): \delta=195.3,194.3,137.3$, 134.6, 133.6, 131.5, 129.1, 60.6, 60.2, 42.7, 38.2, 35.0, 30.1, 30.0, 29.6; MS(EI): m/z(\%) 316(M $\left(\mathrm{M}^{+}\right), 225,211,105(100), 77$; HRMS: calc'd for $\mathrm{C}_{22} \mathrm{H}_{20} \mathrm{O}_{2}$ : 316.1463; found, 316.1459.

2-Cyclohex-2-enyl-1,3-diphenyl-propane-1,3-dione. $\mathrm{IR}(\mathrm{KBr}$ plate): 2931, 2360, 1679, 1643, 1595, 1446, 1269, 971, $709 \mathrm{~cm}^{-1} ;{ }^{1} \mathrm{H}$ $\mathrm{NMR}\left(\mathrm{CDCl}_{3}, 300 \mathrm{MHz}, \mathrm{ppm}\right): \delta=7.99-7.97(\mathrm{~m}, 4 \mathrm{H}), 7.55(\mathrm{~m}, 2 \mathrm{H}), 7.45-$ 7.42(m, 4H), 5.73(m, 1H), 5.52(m, 1H), 5.296(d, 1H, J=9.9Hz), 3.49(m, $1 \mathrm{H}), 2.01(\mathrm{~m}, 2 \mathrm{H}), 1.75(\mathrm{~m}, 2 \mathrm{H}), 1.37(\mathrm{~m}, 2 \mathrm{H}) ;{ }^{13} \mathrm{C} \mathrm{NMR}\left(\mathrm{CDCl}_{3}\right.$,

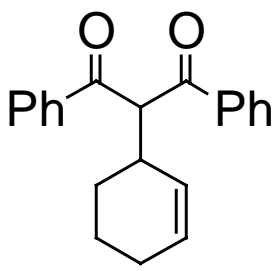
75MHz, ppm): $\delta=201.9,194.9,137.3,133.7,132.0,129.6,128.7,62.8,37.6,27.8,25.5,21.5$; MS(EI): m/z(\%) 304(M $\left.\mathrm{M}^{+}\right), 223,199(100), 105,77$; HRMS: calc'd for $\mathrm{C}_{21} \mathrm{H}_{20} \mathrm{O}_{2:}$ 304.1463; found, 304.1459.

2-Cyclopent-2-enyl-1,3-diphenyl-propane-1,3-dione. $\quad \operatorname{IR}(\mathrm{KBr}$ plate): 2919, 1728, 1695, 1655, 1495, 1265, 987, 693 $\mathrm{cm}^{-1} ;{ }^{1} \mathrm{H} \mathrm{NMR}\left(\mathrm{CDCl}_{3}\right.$, $300 \mathrm{MHz}, \mathrm{ppm}): \delta=8.00-7.93(\mathrm{~m}, 4 \mathrm{H}), 7.59-7.40(\mathrm{~m}, 6 \mathrm{H}), 5.58(\mathrm{~m}, 1 \mathrm{H})$,<smiles>O=C(C(=O)C(c1ccccc1)C1C=CCC1)c1ccccc1</smiles>
$5.18(\mathrm{~d}, 1 \mathrm{H}, \mathrm{J}=9.9 \mathrm{~Hz}), 3.84(\mathrm{~m}, 1 \mathrm{H}), 2.40-2.19(\mathrm{~m}, 4 \mathrm{H}) ;{ }^{13} \mathrm{C} \mathrm{NMR}\left(\mathrm{CDCl}_{3}\right.$, 75MHz, ppm): $\delta=203.5,195.1,133.6,132.5,129.1,128.9,62.2,38.4,32.1,29.3 ; \mathrm{MS}(\mathrm{EI}):$ m/z(\%) 223, 185(100) 105, 77; MS(ESI): m/z 290(M+), 223; HRMS calc'd for $\mathrm{C}_{13} \mathrm{H}_{13} \mathrm{O}\left(\mathrm{M}^{+}-\right.$ $\mathrm{COPh}): 185.0966$; found, 185.0967. 


\section{1,3-Diphenyl-2-(tetrahydro-pyran-2-yl)-propane-1,3-dione.}

$\operatorname{IR}(\mathrm{KBr}$ plate): 2959, 1687, 1653, 1594, 1446, 1196, 1087, 711 $686 \mathrm{~cm}^{-1} ;{ }^{1} \mathrm{H} \mathrm{NMR}\left(\mathrm{CDCl}_{3}, 300 \mathrm{MHz}, \mathrm{ppm}\right): \delta=7.97(\mathrm{~m}, 5 \mathrm{H}), 7.59$ 7.41(m, 5H), 5.48(d, 1H, J=9Hz), 4.93(m, 1H), 3.90(m, 1H), 3.45(m, $1 \mathrm{H}), 1.82(\mathrm{~m}, 2 \mathrm{H}), 1.59(\mathrm{~m}, 2 \mathrm{H}), 1.40(\mathrm{~m}, 2 \mathrm{H}) ;{ }^{13} \mathrm{C} \mathrm{NMR}\left(\mathrm{CDCl}_{3}\right.$,


75MHz, ppm): $\delta=194.63,193.45,149.98,137.51,136.86,133.80,133.38,69.27,63.25$, 36.88, 26.28, 23.71; MS(EI): m/z(\%) 308, 203, 105(100), 77; HRMS calc'd forC ${ }_{20} \mathrm{H}_{18} \mathrm{O}_{3}$ : 308.1399; found, 308.1398 .

\section{2-(6-Methoxy-tetrahydro-pyran-2-yl)-1,3-diphenyl-propane-1,3-}

dione. IR(KBr plate): 2936, 1696, 1596, 1448, 1224, 1119, 1000, 951 $\mathrm{cm}^{-1 ;}{ }^{1} \mathrm{H}-\mathrm{NMR}\left(\mathrm{CDCl}_{3}, 300 \mathrm{MHz}, \mathrm{ppm}\right): \delta=8.04-7.91(\mathrm{~m}, 4 \mathrm{H}), 7.55-$ $7.40(\mathrm{~m}, 6 \mathrm{H}), 5.58(\mathrm{~d}, 1 \mathrm{H}, \mathrm{J}=9.3 \mathrm{~Hz}), 4.50(\mathrm{dt}, 1 \mathrm{H}, \mathrm{J}=9.3,1.7 \mathrm{~Hz}), 4.29(\mathrm{dd}$, $1 \mathrm{H}, \mathrm{J}=8.5,1.3 \mathrm{~Hz}), 3.06(\mathrm{~s}, 3 \mathrm{H}), 1.83-1.76(\mathrm{~m}, 4 \mathrm{H}), 1.66(\mathrm{~m}, 2 \mathrm{H})$; $\mathrm{MS}(\mathrm{EI}): \mathrm{m} / \mathrm{z}(\%) 233,224,184,105(100), 77(100) ;{ }^{13} \mathrm{C} \mathrm{NMR}\left(\mathrm{CDCl}_{3}\right.$,

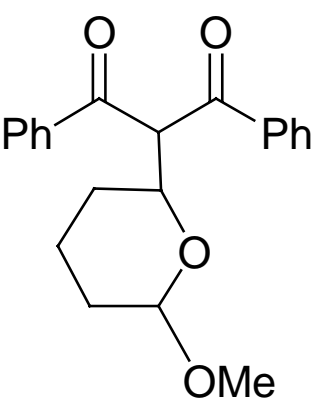
$75 \mathrm{MHz}, \mathrm{ppm}): \delta=194.5,193.3,137.7,133.8,129.0,127.4,98.7,70.2,63.7,54.9,30.2,29.9$, 18.3; $\mathrm{MS}(\mathrm{ESI}): \mathrm{m} / \mathrm{z}(\%) 338.1\left(\mathrm{M}^{+}\right)$; HRMS calc'd for $\mathrm{C}_{14} \mathrm{H}_{17} \mathrm{O}_{3}\left(\mathrm{M}^{+}-\mathrm{COPh}\right): 233.11777$; found, 233.11752. Another diastereoisomer: ${ }^{1} \mathrm{H}-\mathrm{NMR}\left(\mathrm{CDCl}_{3}, 300 \mathrm{MHz}, \mathrm{ppm}\right): \delta=8.02-$ 7.94(m, 4H), 7,56-7,40(m, 6H), 5.43(d, 1H, J=9.3Hz), 4.91(dt, 1H, J=9.3, 1.4Hz), 4.58(m, $1 \mathrm{H}), 3.14(\mathrm{~s}, 3 \mathrm{H}), 1.96-1.79(\mathrm{~m}, 3 \mathrm{H}), 1.68-1.56(\mathrm{~m}, 3 \mathrm{H}) ;{ }^{13} \mathrm{C} \mathrm{NMR}\left(\mathrm{CDCl}_{3}, 75 \mathrm{MHz}, \mathrm{ppm}\right): \delta=$ 194.0, 193.1, 136.9, 134.2, 130.2, 129.0, 128.6, 99.4, 70.8, 64.0, 54.1, 29.6, 29.3, 18.8.

\section{2-(6-Ethyl-tetrahydro-pyran-2-yl)-1,3-diphenyl-propane-1,3-dione.}

IR(KBr plate): 2956, 1704, 1659, 1596, 1498, 1280, 1196, 1029, 760 $\mathrm{cm}^{-1}$; ${ }^{1} \mathrm{H} \mathrm{NMR}\left(\mathrm{CDCl}_{3}, 300 \mathrm{MHz}, \mathrm{ppm}\right): \delta=8.02-7.94(\mathrm{~m}, 4 \mathrm{H}), 7.54-7.40(\mathrm{~m}$, $6 \mathrm{H}), 5.40(\mathrm{~d}, 1 \mathrm{H}, \mathrm{J}=9.8 \mathrm{~Hz}), 4.92(\mathrm{dt}, 1 \mathrm{H}, \mathrm{J}=9.8,2.4 \mathrm{~Hz}), 4.69(\mathrm{~m}, 1 \mathrm{H})$,

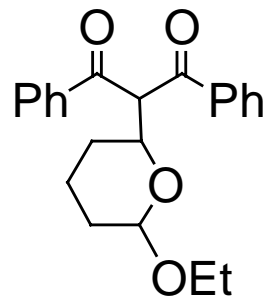
3.41(m, 1H), 3.16(m, 1H), 1.95-1.82(m, 2H), 1.63(m, 2H), 1.41(m, 2H), 1.11(t, 3H, J=8.1, 4.2 $\mathrm{Hz}) ;{ }^{13} \mathrm{C} \mathrm{NMR}\left(\mathrm{CDCl}_{3}, 75 \mathrm{MHz}, \mathrm{ppm}\right): \delta=194.6,193.4,133.9,133.4,129.1,128.8,127.4$, 102.2, 64.1, 62.4, 37.5, 30.1, 29.1, 15.4, 14.6; MS(EI): m/z(\%) 247, 224, 184, 105(100), 77; MS(ESI): m/z(\%) 352.1 ( $\left.\mathrm{M}^{+}\right)$; HRMS calc'd for $\mathrm{C}_{15} \mathrm{H}_{19} \mathrm{O}_{3}\left(\mathrm{M}^{+}-\mathrm{COPh}\right)$ : 247.13342; found, 247.13299. Another diastereoisomer ${ }^{1} \mathrm{H} \mathrm{NMR}\left(\mathrm{CDCl}_{3}, 300 \mathrm{MHz}, \mathrm{ppm}\right): \delta=8.00-7.92(\mathrm{~m}, 4 \mathrm{H})$, 7.59-7.40(m, 6H), 5.58(d, 1H, J=9.9Hz), 4.50(m, 1H), 4.39(dd, 1H, J=8.4, 1.2Hz) 3.35(m, 
$1 \mathrm{H}), 3.22(\mathrm{~m}, 1 \mathrm{H}), 1.86-1.72(\mathrm{~m}, 2 \mathrm{H}), 1.61(\mathrm{~m}, 2 \mathrm{H}) 1.39(\mathrm{~m}, 2 \mathrm{H}), 0,992(\mathrm{t}, 3 \mathrm{H}, \mathrm{J}=8.3,3,9$ $\mathrm{Hz}) ;{ }^{13} \mathrm{C} \mathrm{NMR}\left(\mathrm{CDCl}_{3}, 75 \mathrm{MHz}, \mathrm{ppm}\right): \delta=195.8,194.3,133.1,132.6,130.2,129.8,128.0$, $103.1,63.5,61.6,38.4,30.9,28.3,14.9,14.0$.

2-[6-(1-Benzoyl-2-oxo-2-phenyl-ethyl)-tetrahydro-pyran-2-yl]-1,3diphenyl-propane-1,3-dione. $\operatorname{IR}(\mathrm{KBr}$ plate): 2931, 2344, 1696, 1559, $1450,1273,1192,762 \mathrm{~cm}^{-1} ;{ }^{1} \mathrm{H} \mathrm{NMR}\left(\mathrm{CDCl}_{3}, 300 \mathrm{MHz}, \mathrm{ppm}\right): \delta=7.89(\mathrm{~m}$, $4 \mathrm{H}), 7.66(\mathrm{~m}, 4 \mathrm{H}), 7.49-7.28(\mathrm{~m}, 12 \mathrm{H}), 5.27(\mathrm{~d}, 2 \mathrm{H}, \mathrm{J}=9 \mathrm{~Hz}), 4.52(\mathrm{~m}, 2 \mathrm{H})$, $1.82(\mathrm{~m}, 3 \mathrm{H}), 1.36(\mathrm{~m}, 3 \mathrm{H}) ;{ }^{13} \mathrm{C} \mathrm{NMR}\left(\mathrm{CDCl}_{3}, 75 \mathrm{MHz}, \mathrm{ppm}\right): \delta=195.2$, $189.2,133.7,133.3,129.1,128.6,79.2,74.2,63.9,33.1,30.1,29.0$,

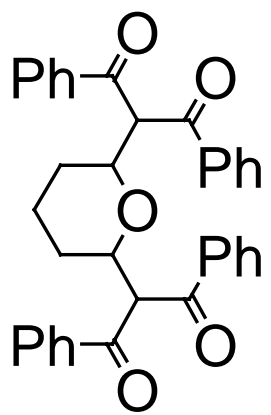
27.0, 23.1; MS(EI): m/z(\%) 306, 223, 146, 105(100), 77; MS(ESI): m/z (\%) 530(M+), 307; HRMS calc'd for $\mathrm{C}_{20} \mathrm{H}_{18} \mathrm{O}_{3}\left(\mathrm{M}^{+}\right.$-dibenzoylmethane): 306.12559; found, 306.12531 .

1,3-Diphenyl-2-(tetrahydro-furan-2-yl)-propane-1,3-dione. IR( $\mathrm{KBr}$ plate): $2943,2319,1691,1603,1442,1289,1072,818,685 \mathrm{~cm}^{-1} ;{ }^{1} \mathrm{H}-$ $\operatorname{NMR}\left(\mathrm{CDCl}_{3}, 300 \mathrm{MHz}, \mathrm{ppm}\right): \delta=8.02(\mathrm{~m}, 4 \mathrm{H}), 7.53-7.41(\mathrm{~m}, 6 \mathrm{H})$, 5.42(d, $1 \mathrm{H}, \mathrm{J}=8.1 \mathrm{~Hz}), 4.83(\mathrm{~m}, 1 \mathrm{H}), 3.83-3.69(\mathrm{~m}, 2 \mathrm{H}), 2.29(\mathrm{~m}, 2 \mathrm{H})$, $1.95(\mathrm{~m}, 2 \mathrm{H}) ;{ }^{13} \mathrm{C} \mathrm{NMR}\left(\mathrm{CDCl}_{3}, 75 \mathrm{MHz}, \mathrm{ppm}\right): \delta=194.7,194.3,137.0$,

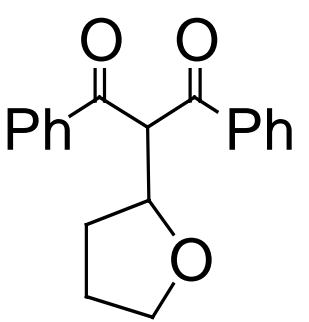
130.6, 138.8, 133.6, 129.0, 79.7, 68.4, 63.0, 30.8, 25.9; MS(EI) m/z(\%): 224, 189, 105(100), 77; MS(ESI): m/z (\%) $294.1\left(\mathrm{M}^{+}\right)$; HRMS calc'd for $\mathrm{C}_{12} \mathrm{H}_{13} \mathrm{O}_{2}\left(\mathrm{M}^{+}-\mathrm{COPh}\right)$ : 189.09155; found: 189.09171 .

2-Cyclooct-2-enyl-1-phenyl-butane-1,3-dione. One of the diastereoisomer can be partially isolated using crystallization. $\mathrm{IR}(\mathrm{KBr}$ plate): $2923,2855,2360,1708,1660,1447,1354,970 \mathrm{~cm}^{-1} ;{ }^{1} \mathrm{H}$ $\mathrm{NMR}\left(\mathrm{CDCl}_{3}, 300 \mathrm{MHz}, \mathrm{ppm}\right): \delta=8.00-7.97(\mathrm{~m}, 2 \mathrm{H}), 7.57-7.43(\mathrm{~m}, 3 \mathrm{H})$, 5.63(m, 1H), 5.17(m, 1H), 4.58(d, 1H, J=9.1Hz), 3.61(m, 1H), 2.40(m,

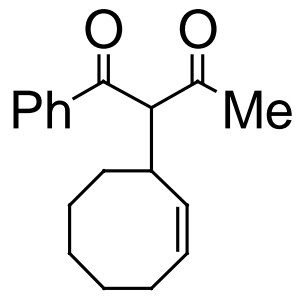
2H), 2.21(s, 3H), 2.11(m, 2H), 1.74(m, 2H), 1.49(m, 4H); $\left.{ }^{13} \mathrm{C} \mathrm{NMR(CDCl} 3,75 \mathrm{MHz}, \mathrm{ppm}\right)$ : $\delta=204.4,195.6,133.8,132.1,130.4,129.0,70.3,37.5,33.8,29.8,28.1,27.3,25.6$; MS(EI): $\mathrm{m} / \mathrm{z}(\%) 270\left(\mathrm{M}^{+}\right), 227(100), 165,105,77$; HRMS calc'd for $\mathrm{C}_{18} \mathrm{H}_{22} \mathrm{O}_{2:}$ 270.1620; found, 270.1616 . 
Another diastereoisomer (from the mixture and some peaks are difficult to distinguished): ${ }^{1} \mathrm{H} \mathrm{NMR}\left(\mathrm{CDCl}_{3}, 300 \mathrm{MHz}, \mathrm{ppm}\right): \delta=4.41(\mathrm{~d}, 1 \mathrm{H}, \mathrm{J}=8.6 \mathrm{~Hz}), 3.46(\mathrm{~m}, 1 \mathrm{H})$, 2.10(s, 3H); ${ }^{13} \mathrm{C} \mathrm{NMR}\left(\mathrm{CDCl}_{3}, 75 \mathrm{MHz}, \mathrm{ppm}\right): \delta=203.6,194.0,135.4,69.6,36.8,34.3,26.8$, 24.5.

1-Phenyl-2-(tetrahydro-pyran-2-yl)-butane-1,3-dione. one of the $\mathrm{Ph}_{\mathrm{Me}}$ diastereoisomer can be partially isolated using a mixture of 5:1:1(hexanes, ethyl acetate, ether) on an analytical TLC. $\mathrm{IR}(\mathrm{KBr}$ plate): 2936, 2348,

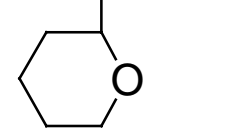
$1708,1656,1555,1450,1233,976 \mathrm{~cm}^{-1} ;{ }^{1} \mathrm{H} \mathrm{NMR}\left(\mathrm{CDCl}_{3}, 300 \mathrm{MHz}, \mathrm{ppm}\right): \delta=8.03(\mathrm{~m}, 2 \mathrm{H})$, 7.59-7.45(m, 3H), 4.64(d, 1H, J=9.6Hz), 4.28(m, 1H), 4.01(m, 1H), 3.49(m, 1H), 2.24(s, 3H), 1.82(m, 1H), 1.59(m, 5H). ${ }^{13} \mathrm{C} \mathrm{NMR}\left(\mathrm{CDCl}_{3}, 75 \mathrm{MHz}, \mathrm{ppm}\right): 202.8,193.8,150.0,136.9$, 134.1, 133.9, 129.1, 70.3, 59.8, 30.5, 27.9, 26.1, 23.5; MS(EI): m/z(\%) 203, 147, 105(100), 77; MS(ESI): m/z(\%) $246.1\left(\mathrm{M}^{+}\right)$; HRMS calc'd for $\mathrm{C}_{13} \mathrm{H}_{15} \mathrm{O}_{2}\left(\mathrm{M}^{+}-\mathrm{Ac}\right)$ : 203.10720; found: 203.10752. Another diastereoisomer (from the mixture and some peaks are difficult to distinguished): ${ }^{1} \mathrm{H} \mathrm{NMR}\left(\mathrm{CDCl}_{3}, 300 \mathrm{MHz}, \mathrm{ppm}\right): 4.69(\mathrm{~d}, 1 \mathrm{H}, \mathrm{J}=9.6 \mathrm{~Hz}), 3.82(\mathrm{~m}, 1 \mathrm{H}), 2.18(\mathrm{~s}$, $3 \mathrm{H}) ;{ }^{13} \mathrm{C} \mathrm{NMR}\left(\mathrm{CDCl}_{3}, 75 \mathrm{MHz}, \mathrm{ppm}\right): 203.1,192.2$, 148.8, 69.1, 60.4, 31.4, 28.5, 24.0.

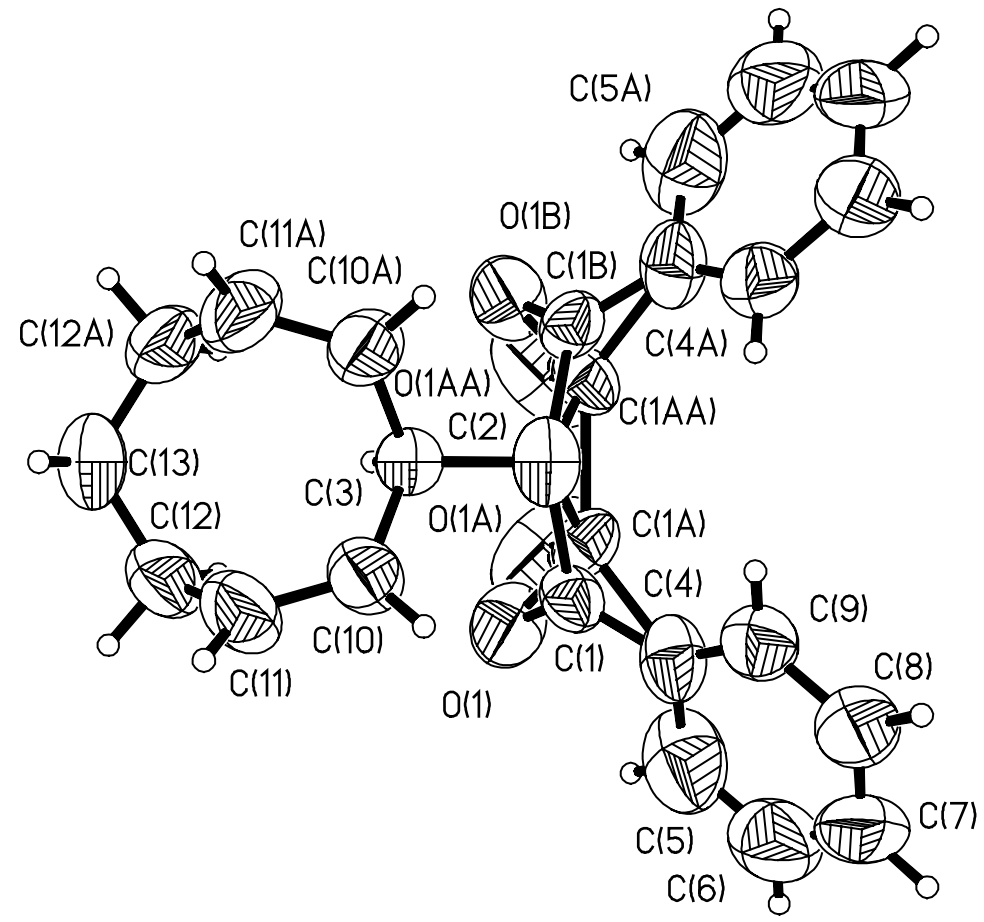


Table 1. Crystal data and structure refinement for 1 .

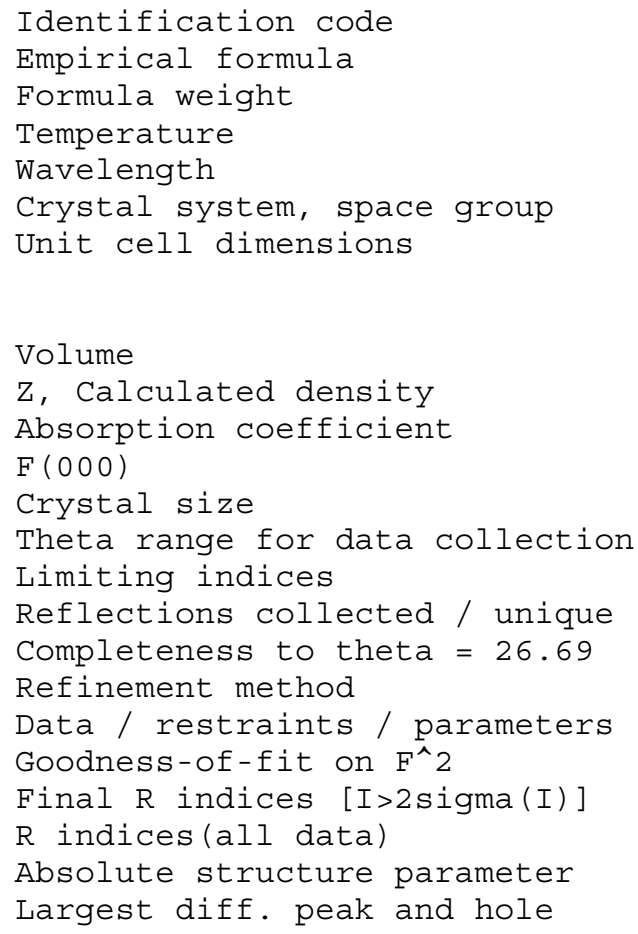

1

C11.50 H11 O

165.20

$571(2) \mathrm{K}$

$0.71073 \mathrm{~A}$

orthorhomic, Pmn21

$\mathrm{a}=17.307(14) \mathrm{A}$ alpha $=90 \mathrm{deg}$.

$\mathrm{b}=5.500(5) \mathrm{A}$ beta $=90 \mathrm{deg}$.

$\mathrm{c}=9.735(8) \mathrm{A}$ gamma $=90 \mathrm{deg}$.

$926.6(13) \mathrm{A}^{\wedge} 3$

$4,1.184 \mathrm{Mg} / \mathrm{m}^{\wedge} 3$

$0.074 \mathrm{~mm}^{\wedge}-1$

352

$.4 \times .2 \times .2 \mathrm{~mm}$

3.70 to 26.69 deg.

$-21<=\mathrm{h}<=21, \quad-6<=\mathrm{k}<=6,-6<=1<=12$

$4302 / 1348$ [R(int) $=0.1328]$

$94.9 \%$

Full-matrix least-squares on $\mathrm{F}^{\wedge} 2$

$1348 / 1 / 138$

1.013

$\mathrm{R} 1=0.0722, \mathrm{wR} 2=0.2040$

$\mathrm{R} 1=0.0959, \mathrm{wR} 2=0.2295$

$-1(5)$

0.255 and -0.290 e. $A^{\wedge}-3$ 
Table 2. Atomic coordinates $\left(\mathrm{x} 10^{\wedge} 4\right)$ and equivalent isotropic displacement parameters $\left(A^{\wedge} 2 \times 10^{\wedge} 3\right)$ for 1 .

$\mathrm{U}(\mathrm{eq})$ is defined as one third of the trace of the orthogonalized Uij tensor.

\begin{tabular}{|c|c|c|c|c|}
\hline & $x$ & $\mathrm{y}$ & $z$ & $\mathrm{U}(\mathrm{eq})$ \\
\hline$O(1)$ & $1045(6)$ & $-712(15)$ & $925(8)$ & $119(3)$ \\
\hline$C(1)$ & $865(6)$ & $1189(18)$ & $402(10)$ & $72(2)$ \\
\hline$C(2)$ & 0 & $2376(9)$ & 849 (6) & $76(2)$ \\
\hline$C(3)$ & 0 & $2299(7)$ & $2399(5)$ & $61(1)$ \\
\hline$C(4)$ & $1265(3)$ & $2209(8)$ & $-659(5)$ & $86(1)$ \\
\hline$C(5)$ & $1949(5)$ & $1008(14)$ & $-965(8)$ & $136(3)$ \\
\hline$C(6)$ & $2453(4)$ & $1914(18)$ & $-1901(9)$ & $131(2)$ \\
\hline$C(7)$ & $2309(3)$ & $3937(15)$ & $-2579(8)$ & $126(2)$ \\
\hline$C(8)$ & $1635(3)$ & $5235(9)$ & $-2300(7)$ & $109(2)$ \\
\hline$C(9)$ & $1129(3)$ & $4317(9)$ & $-1345(5)$ & $87(1)$ \\
\hline$C(10)$ & $706(2)$ & $3467(8)$ & $2984(6)$ & $88(1)$ \\
\hline$C(11)$ & $927(3)$ & $3071(11)$ & $4335(6)$ & $106(2)$ \\
\hline$C(12)$ & $754(3)$ & $778(10)$ & $5059(6)$ & $99(2)$ \\
\hline$C(13)$ & 0 & $697(14)$ & $5884(8)$ & $98(2)$ \\
\hline$C(1 \mathrm{~A})$ & $502(7)$ & $940(20)$ & $187(14)$ & $57(3)$ \\
\hline$O(1 A)$ & $528(7)$ & $-1248(12)$ & $383(14)$ & $113(4)$ \\
\hline
\end{tabular}


Table 3. Bond lengths [A] and angles [deg] for 1 .

\begin{tabular}{|c|c|}
\hline $\mathrm{O}(1)-\mathrm{C}(1)$ & $1.204(11)$ \\
\hline$C(1)-C(4)$ & $1.364(12)$ \\
\hline$C(1)-C(2)$ & $1.690(10)$ \\
\hline$C(2)-C(3)$ & $1.509(8)$ \\
\hline$C(2)-C(1) \# 1$ & $1.690(10)$ \\
\hline$C(3)-C(10)$ & $1.493(5)$ \\
\hline$C(3)-C(10) \# 1$ & $1.493(5)$ \\
\hline$C(4)-C(9)$ & $1.358(7)$ \\
\hline$C(4)-C(5)$ & $1.388(9)$ \\
\hline$C(5)-C(6)$ & $1.357(11)$ \\
\hline$C(6)-C(7)$ & $1.317(9)$ \\
\hline$C(7)-C(8)$ & $1.395(9)$ \\
\hline$C(8)-C(9)$ & $1.373(8)$ \\
\hline$C(10)-C(11)$ & $1.387(8)$ \\
\hline$C(11)-C(12)$ & $1.476(8)$ \\
\hline$C(12)-C(13)$ & $1.533(7)$ \\
\hline$C(13)-C(12) \# 1$ & $1.533(7)$ \\
\hline$C(1 A)-O(1 A)$ & $1.222(14)$ \\
\hline$C(1 A)-C(1 A) \# 1$ & $1.74(2)$ \\
\hline$O(1 A)-O(1 A) \# 1$ & $1.83(2)$ \\
\hline $\mathrm{O}(1)-\mathrm{C}(1)-\mathrm{C}(4)$ & $123.1(8)$ \\
\hline$O(1)-C(1)-C(2)$ & $117.1(9)$ \\
\hline$C(4)-C(1)-C(2)$ & $119.1(6)$ \\
\hline$C(3)-C(2)-C(1) \# 1$ & $104.3(4)$ \\
\hline$C(3)-C(2)-C(1)$ & $104.3(4)$ \\
\hline$C(1) \# 1-C(2)-C(1)$ & $124.7(8)$ \\
\hline$C(10)-C(3)-C(10) \# 1$ & $109.8(5)$ \\
\hline$C(10)-C(3)-C(2)$ & $111.7(3)$ \\
\hline$C(10) \# 1-C(3)-C(2)$ & $111.7(3)$ \\
\hline$C(9)-C(4)-C(1)$ & $129.4(5)$ \\
\hline$C(9)-C(4)-C(5)$ & $116.6(6)$ \\
\hline$C(1)-C(4)-C(5)$ & $113.6(6)$ \\
\hline$C(6)-C(5)-C(4)$ & $121.2(7)$ \\
\hline$C(7)-C(6)-C(5)$ & $121.7(6)$ \\
\hline$C(6)-C(7)-C(8)$ & $119.6(6)$ \\
\hline$C(9)-C(8)-C(7)$ & $118.5(5)$ \\
\hline$C(4)-C(9)-C(8)$ & $122.5(5)$ \\
\hline$C(11)-C(10)-C(3)$ & $121.3(4)$ \\
\hline$C(10)-C(11)-C(12)$ & $122.1(5)$ \\
\hline$C(11)-C(12)-C(13)$ & $116.6(5)$ \\
\hline$C(12)-C(13)-C(12) \# 1$ & $116.6(6)$ \\
\hline$O(1 A)-C(1 A)-C(1 A) \# 1$ & $92.1(7)$ \\
\hline$C(1 A)-O(1 A)-O(1 A) \# 1$ & $87.9(7)$ \\
\hline
\end{tabular}

Symmetry transformations used to generate equivalent atoms: \#1 $-\mathrm{x}, \mathrm{y}, \mathrm{z}$ 
Table 4. Anisotropic displacement parameters $\left(A^{\wedge} 2 \times 10^{\wedge} 3\right)$ for 1 . The anisotropic displacement factor exponent takes the form: $-2 \mathrm{pi}^{\wedge} 2$ [ h^2 $\left.\mathrm{a}^{\star \wedge} 2 \mathrm{U11}+\ldots+2 \mathrm{~h} \mathrm{k} a * \mathrm{~b} * \mathrm{U} 12\right]$

\begin{tabular}{|c|c|c|c|c|c|c|}
\hline & U11 & $\mathrm{U} 22$ & U33 & $\mathrm{U} 23$ & U13 & U12 \\
\hline$O(1)$ & $154(6)$ & $100(5)$ & $103(5)$ & $28(4)$ & $30(5)$ & $66(5)$ \\
\hline$C(1)$ & $77(6)$ & $77(5)$ & $62(5)$ & $-2(4)$ & $-4(5)$ & $25(5)$ \\
\hline$C(2)$ & $120(4)$ & $48(2)$ & $62(3)$ & $0(2)$ & 0 & 0 \\
\hline$C(3)$ & $66(3)$ & $53(2)$ & $66(3)$ & $2(2)$ & 0 & 0 \\
\hline$C(4)$ & $112(3)$ & $87(3)$ & $59(2)$ & $-7(2)$ & $0(2)$ & $14(2)$ \\
\hline$C(5)$ & $165(6)$ & $148(5)$ & $95(4)$ & $-13(4)$ & $4(4)$ & $60(4)$ \\
\hline$C(6)$ & $95(4)$ & $187(7)$ & $110(5)$ & $-26(5)$ & $-7(4)$ & $18(4)$ \\
\hline$C(7)$ & $74(3)$ & $188(6)$ & $116(5)$ & $-11(5)$ & $-7(3)$ & $-37(3)$ \\
\hline$C(8)$ & $100(3)$ & $120(3)$ & $107(4)$ & $23(3)$ & $-8(3)$ & $-31(3)$ \\
\hline$C(9)$ & $77(2)$ & $97(3)$ & $87(3)$ & $8(2)$ & $-7(2)$ & $-7(2)$ \\
\hline$C(10)$ & $79(2)$ & $96(3)$ & $87(3)$ & $21(2)$ & $-13(2)$ & $-21(2)$ \\
\hline$C(11)$ & $98(3)$ & $127(4)$ & $92(3)$ & $24(3)$ & $-33(3)$ & $-41(3)$ \\
\hline C (12) & $83(3)$ & $124(3)$ & $89(3)$ & $26(3)$ & $-21(2)$ & $1(3)$ \\
\hline$C(13)$ & $114(5)$ & $105(4)$ & $75(4)$ & $16(3)$ & 0 & 0 \\
\hline$C(1 A)$ & $54(7)$ & $60(5)$ & $57(7)$ & $-11(4)$ & $8(6)$ & $0(5)$ \\
\hline$O(1 \mathrm{~A})$ & $141(8)$ & $43(3)$ & $156(10)$ & $-8(5)$ & $64(8)$ & $11(4)$ \\
\hline
\end{tabular}


Table 5. Hydrogen coordinates $\left(x 0^{\wedge} 4\right)$ and isotropic displacement parameters $\left(A^{\wedge} 2 \times 10^{\wedge} 3\right)$ for 1 .

\begin{tabular}{lrrrc}
\hline & & & & \\
& & $Y$ & & $U(e q)$ \\
\hline$H(2 A)$ & 0 & 4089 & 573 & $150(40)$ \\
$H(3 A)$ & 0 & 588 & 2681 & 74 \\
$H(5 A)$ & 2064 & -444 & -520 & 163 \\
$H(6 A)$ & 2912 & 1087 & -2069 & 157 \\
$H(7 A)$ & 2655 & 4497 & -3238 & 151 \\
$H(8 A)$ & 1529 & 6688 & -2752 & 131 \\
$H(9 A)$ & 677 & 5169 & -1160 & 104 \\
$H(10 A)$ & 1003 & 4481 & 2431 & 105 \\
$H(11 A)$ & 1192 & 433 & 4799 & 127 \\
$H(12 A)$ & 1178 & -521 & 5680 & 118 \\
$H(12 B)$ & 739 & 2058 & 4385 & 118 \\
$H(13 A)$ & 0 & -778 & 6518 & 118 \\
$H(13 B)$ & 0 & & 6430 & \\
\hline
\end{tabular}



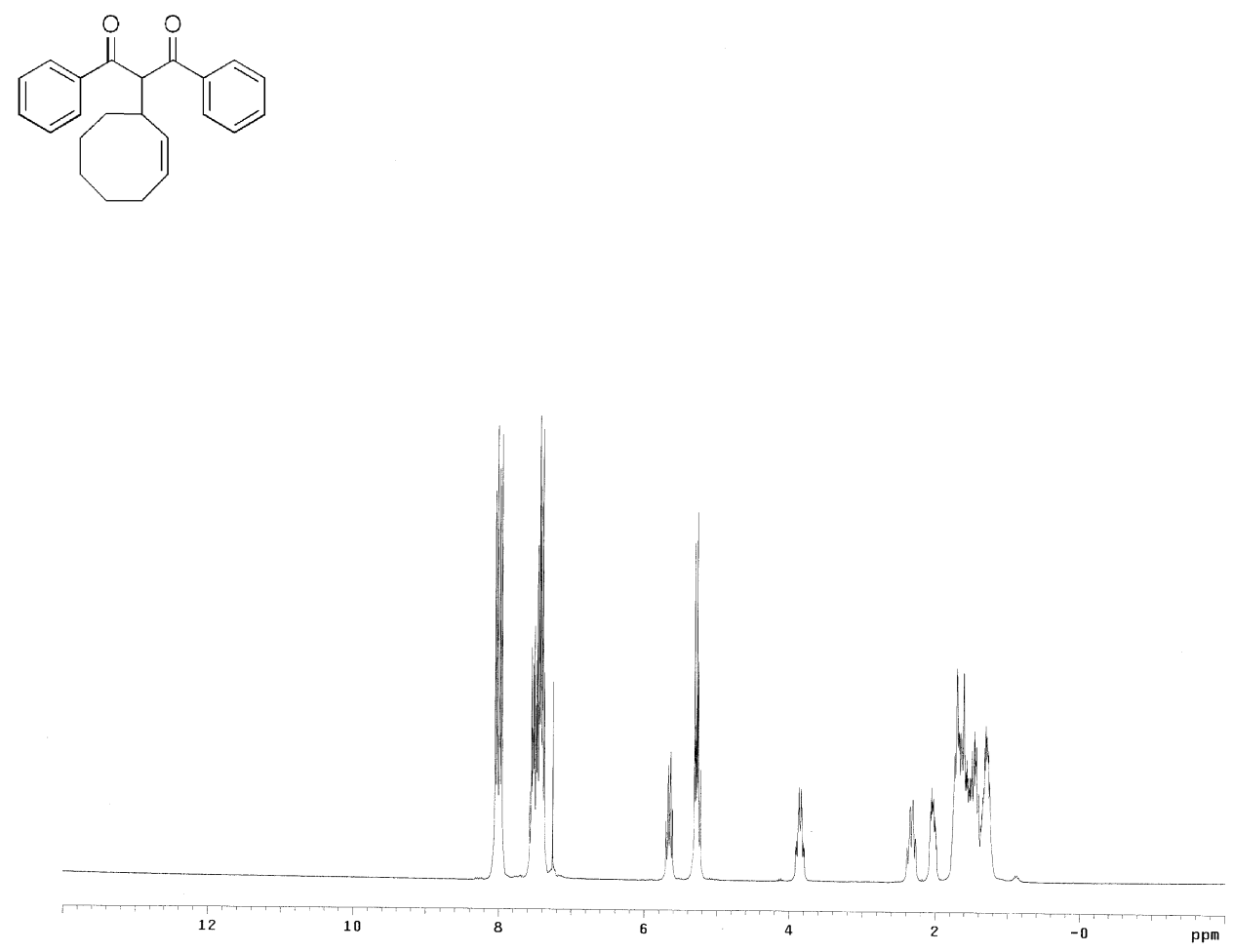


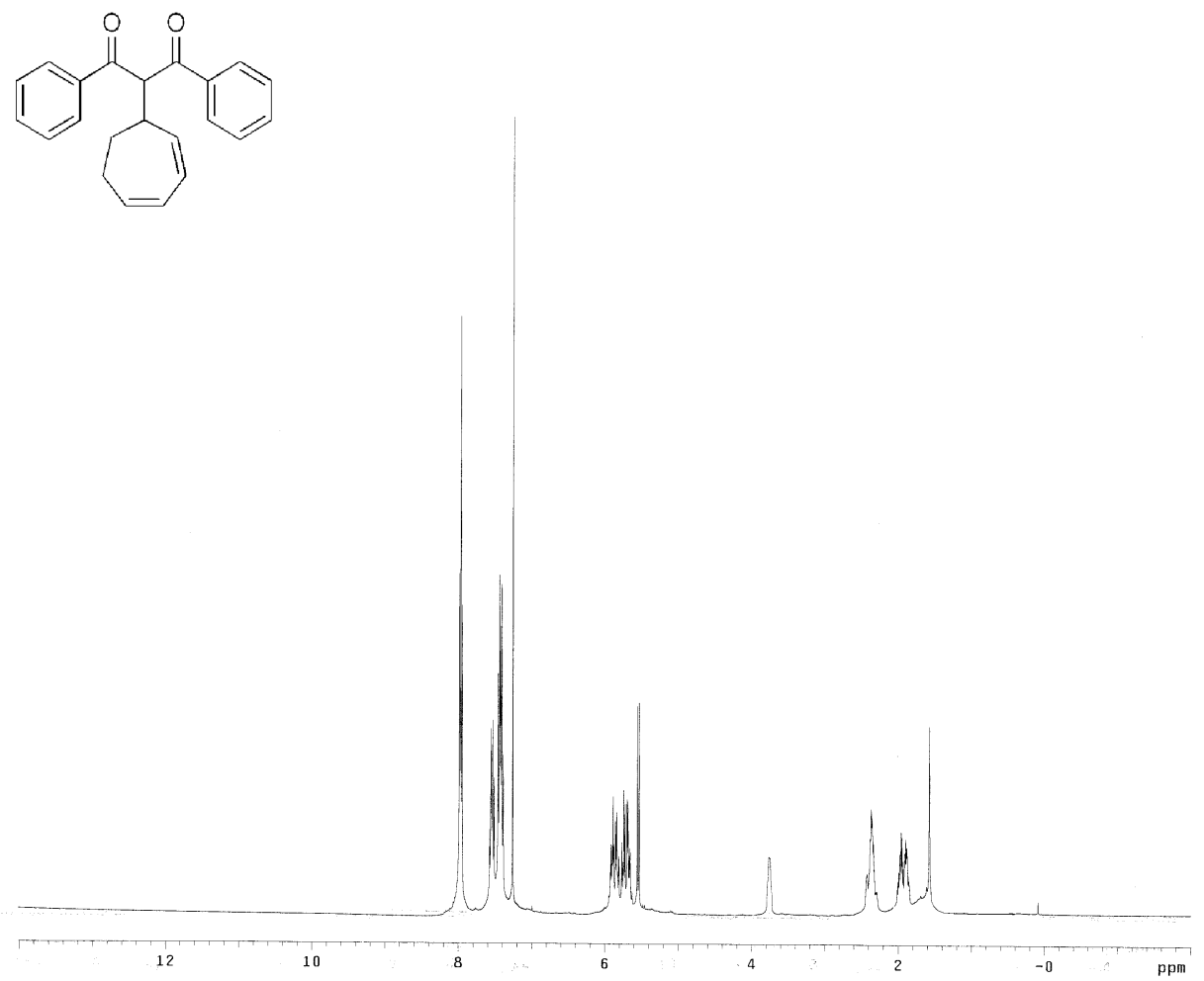

S12 


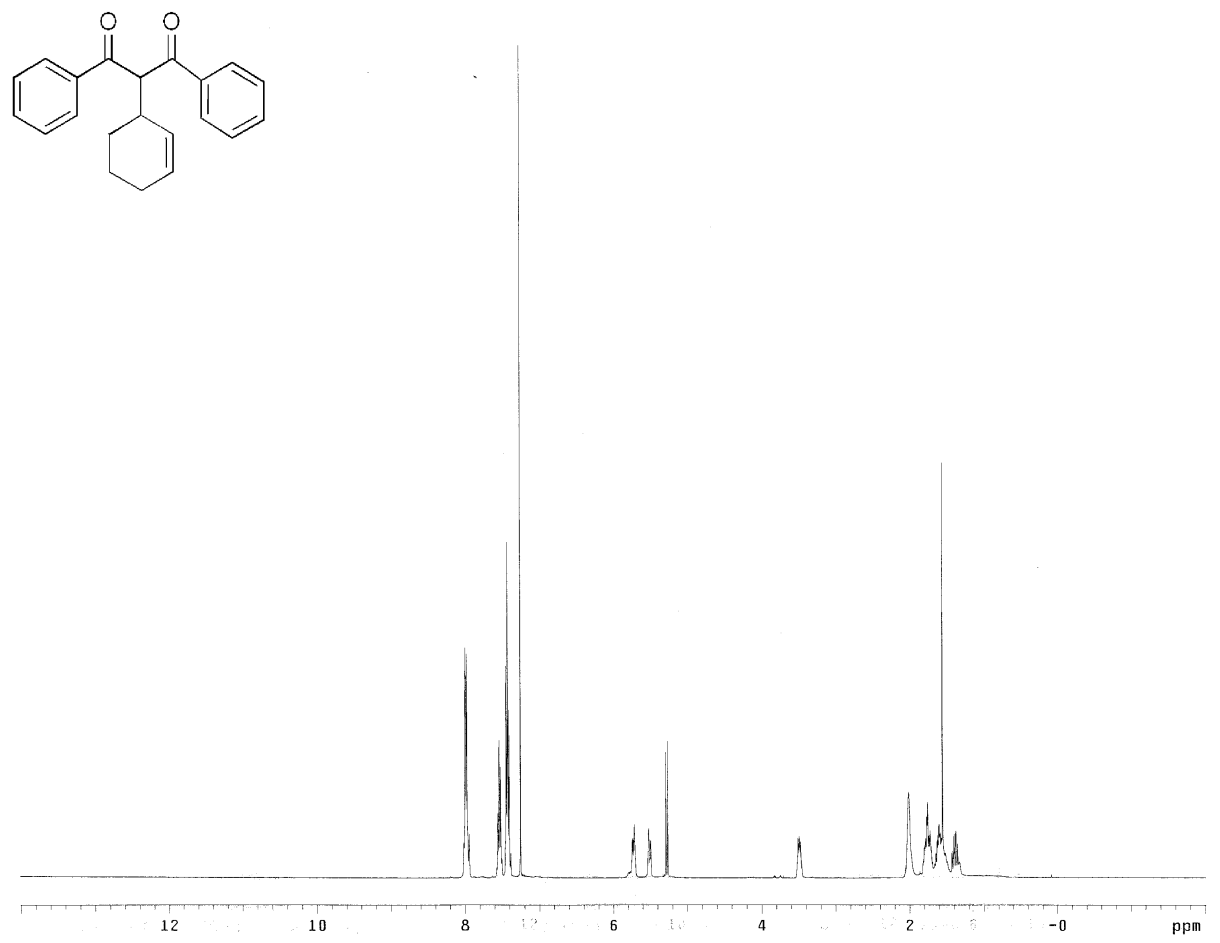

S13 


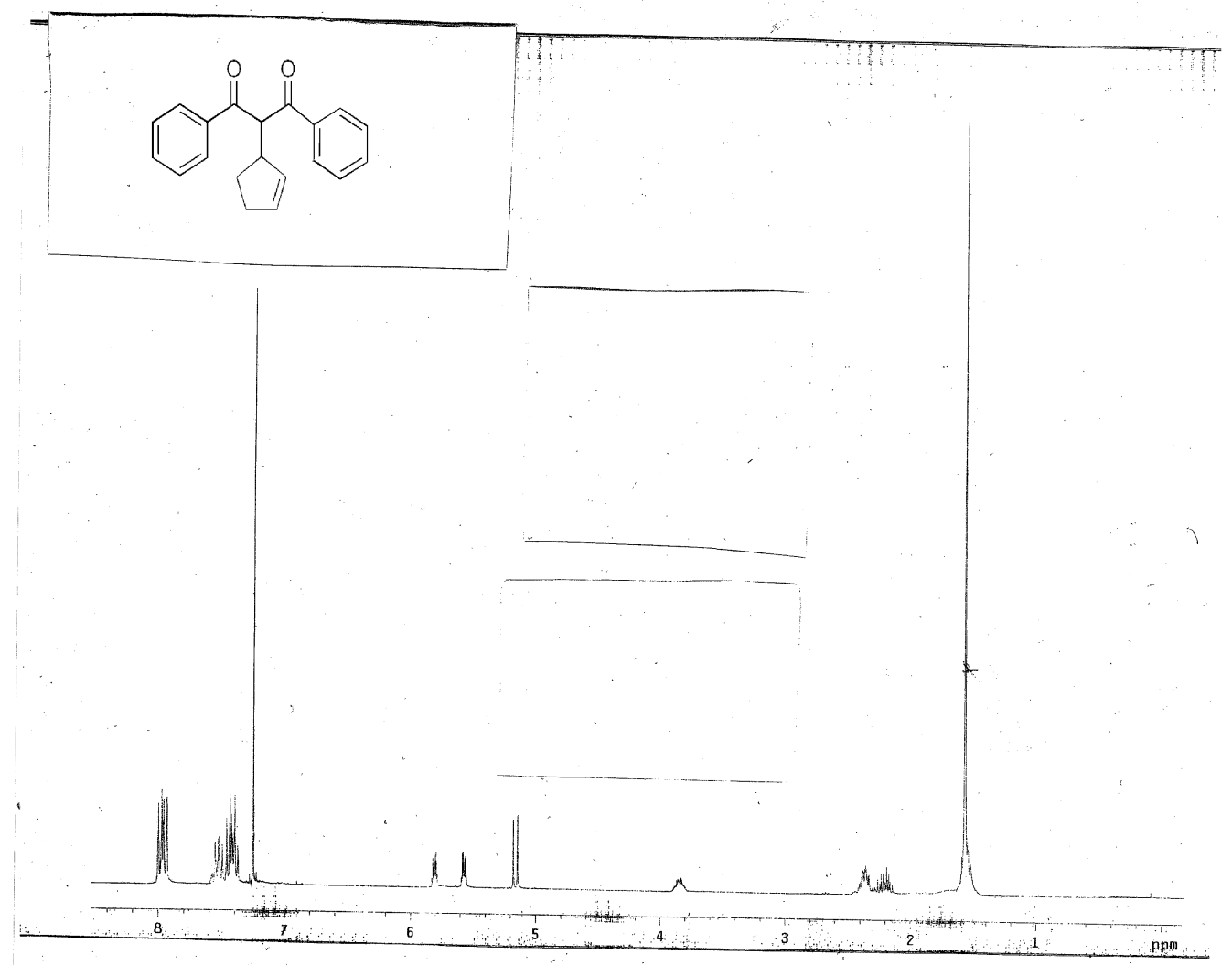



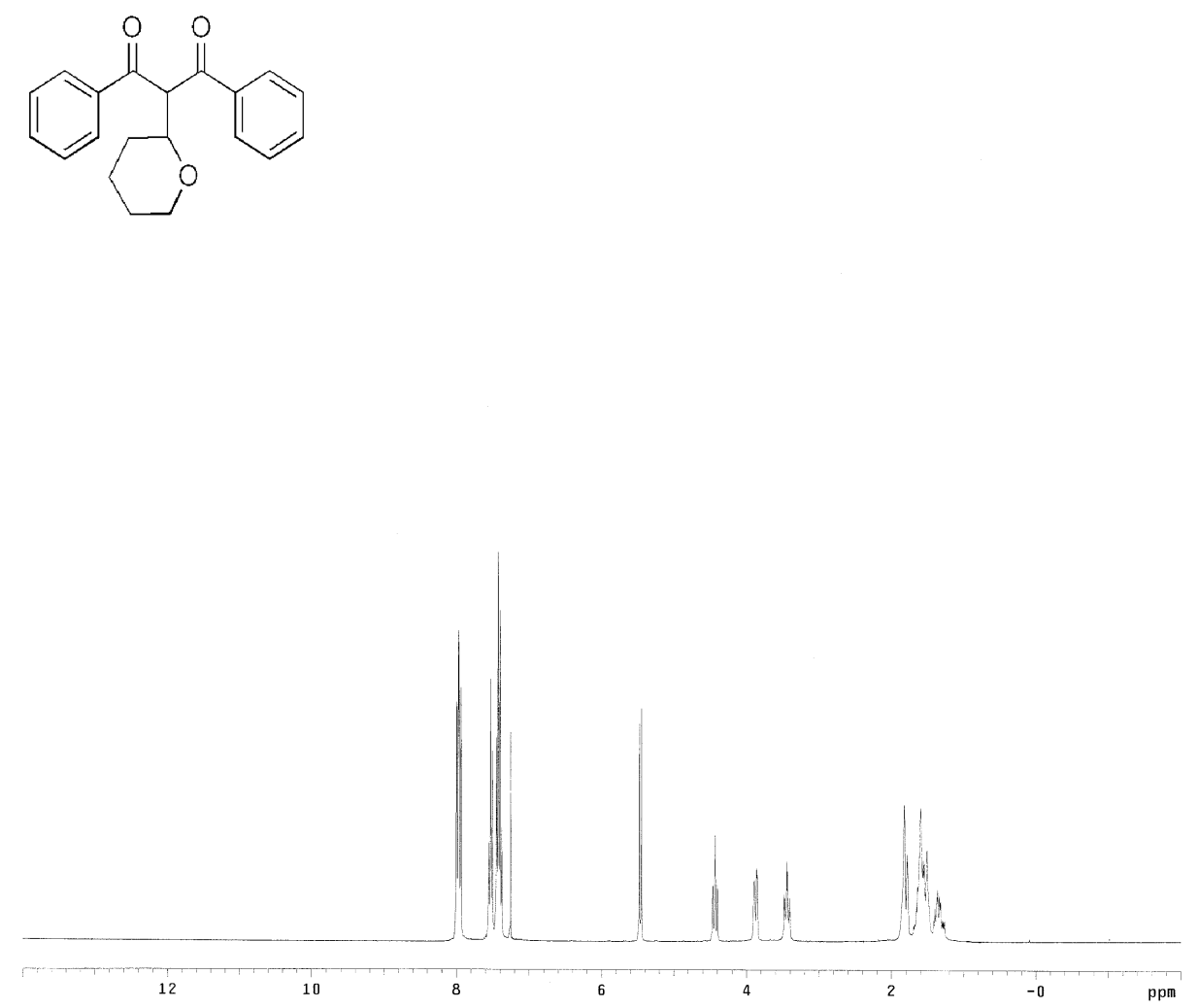

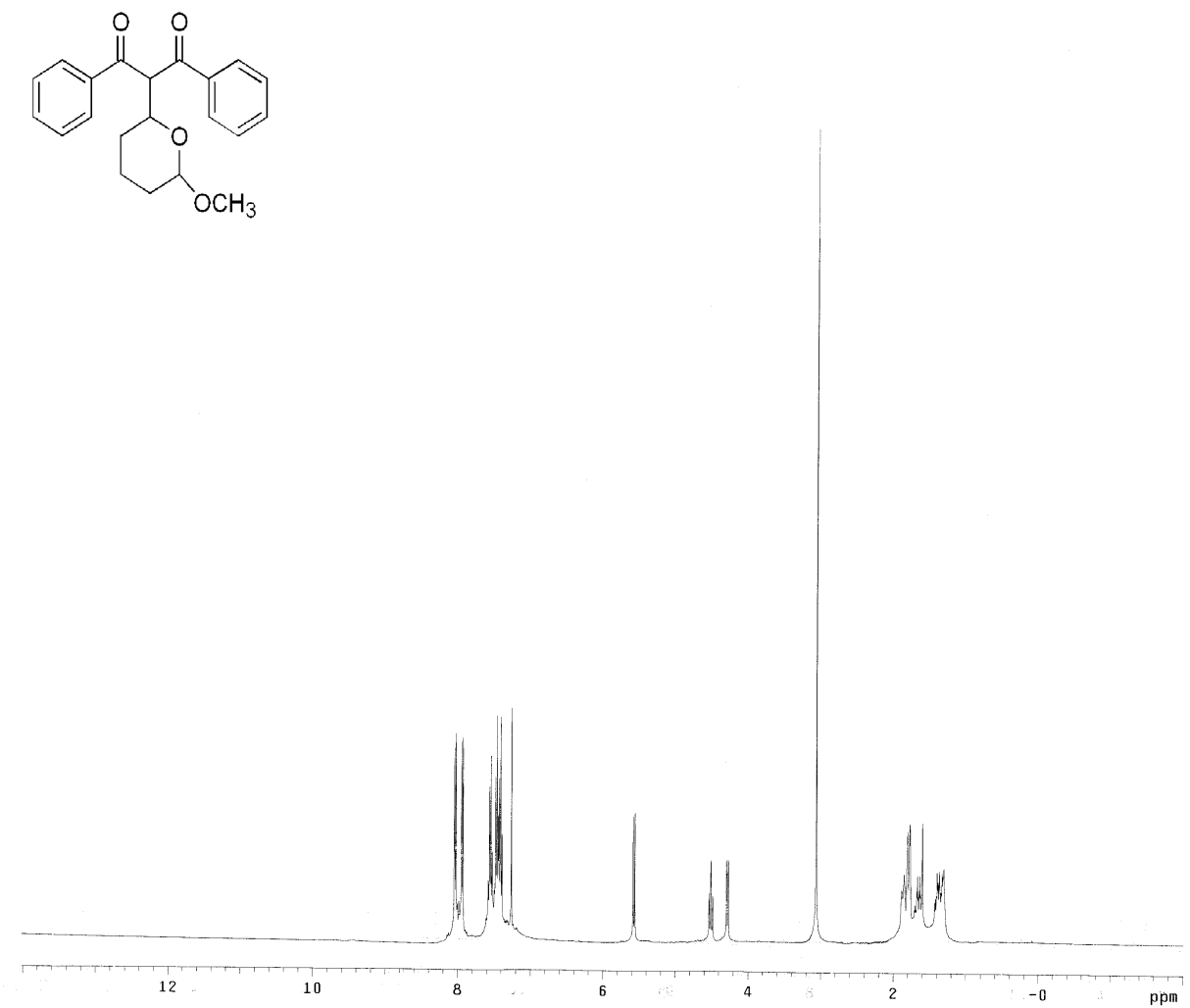

S16 


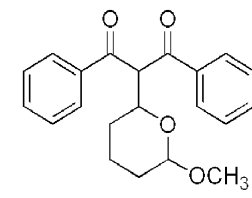

Another diastereoisomer

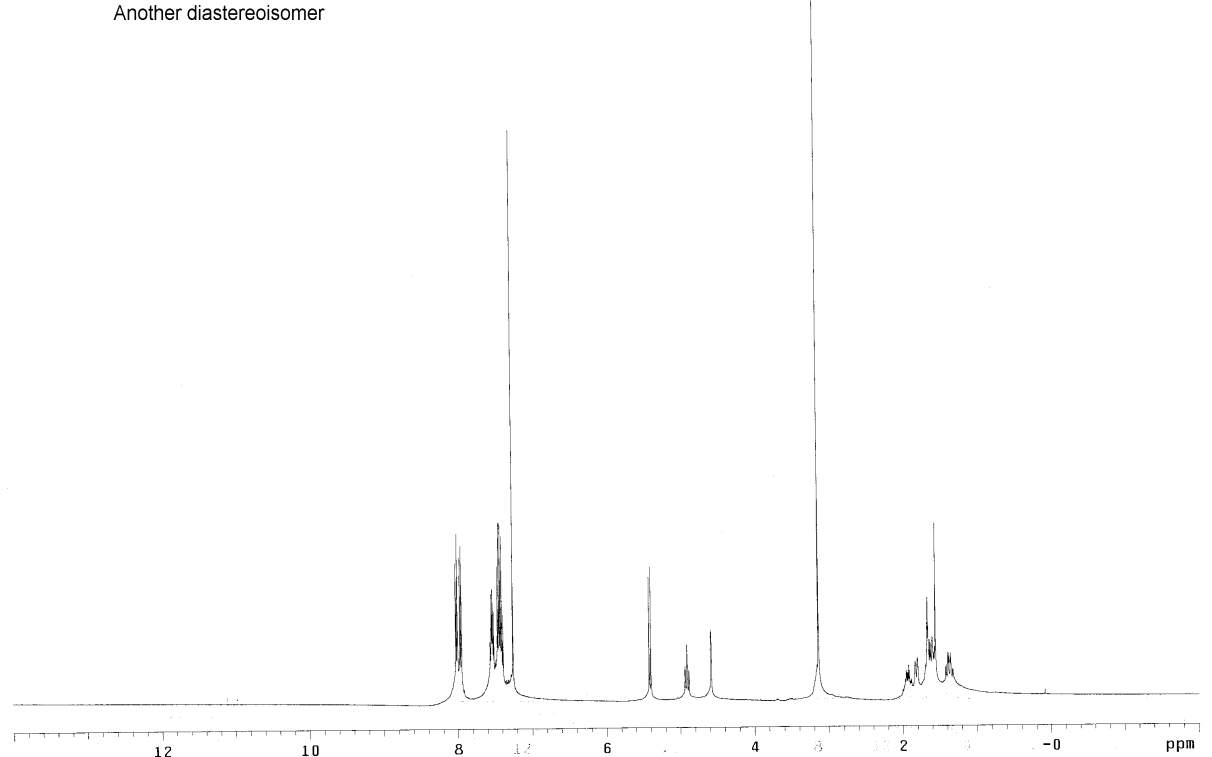




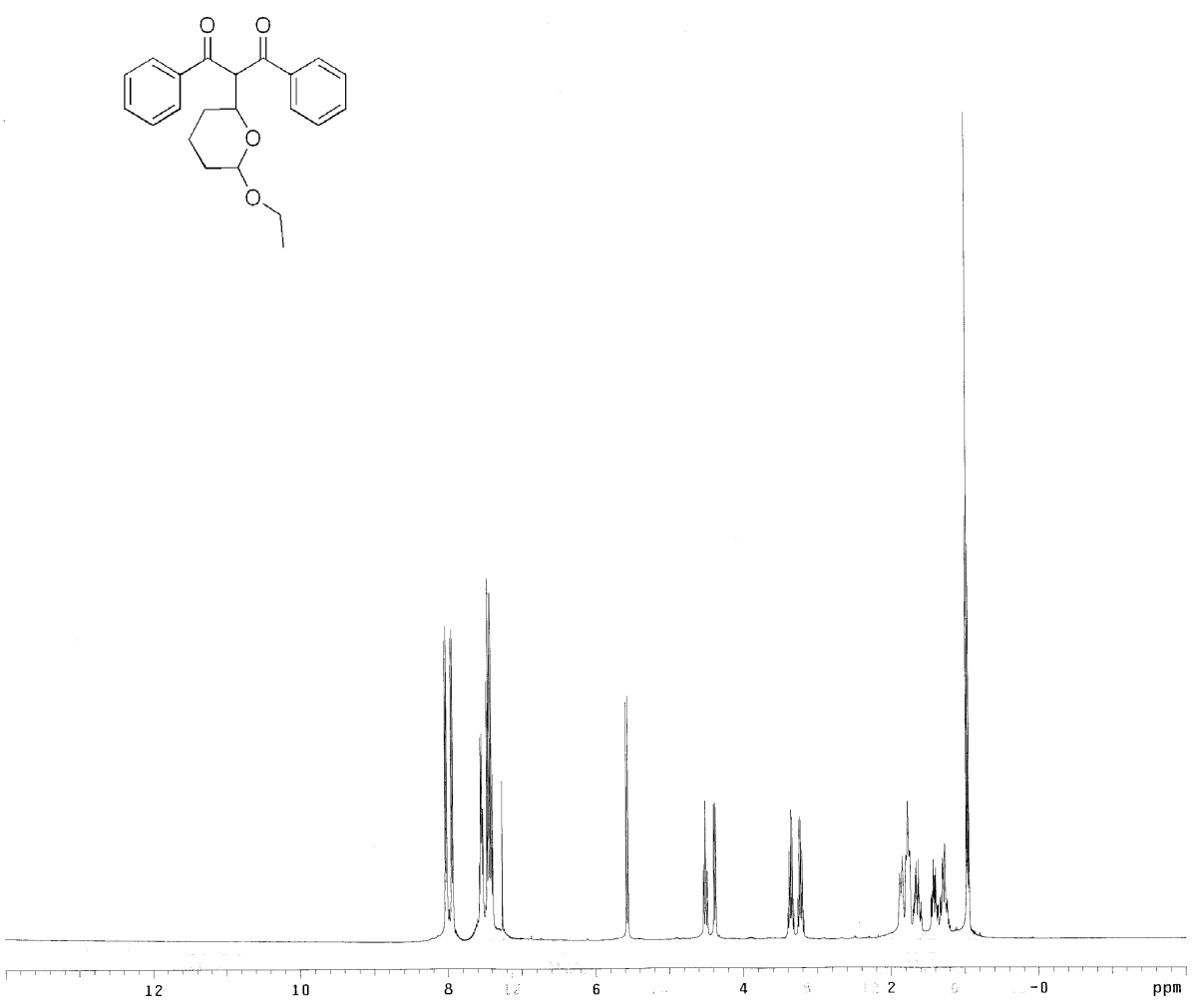

S18 


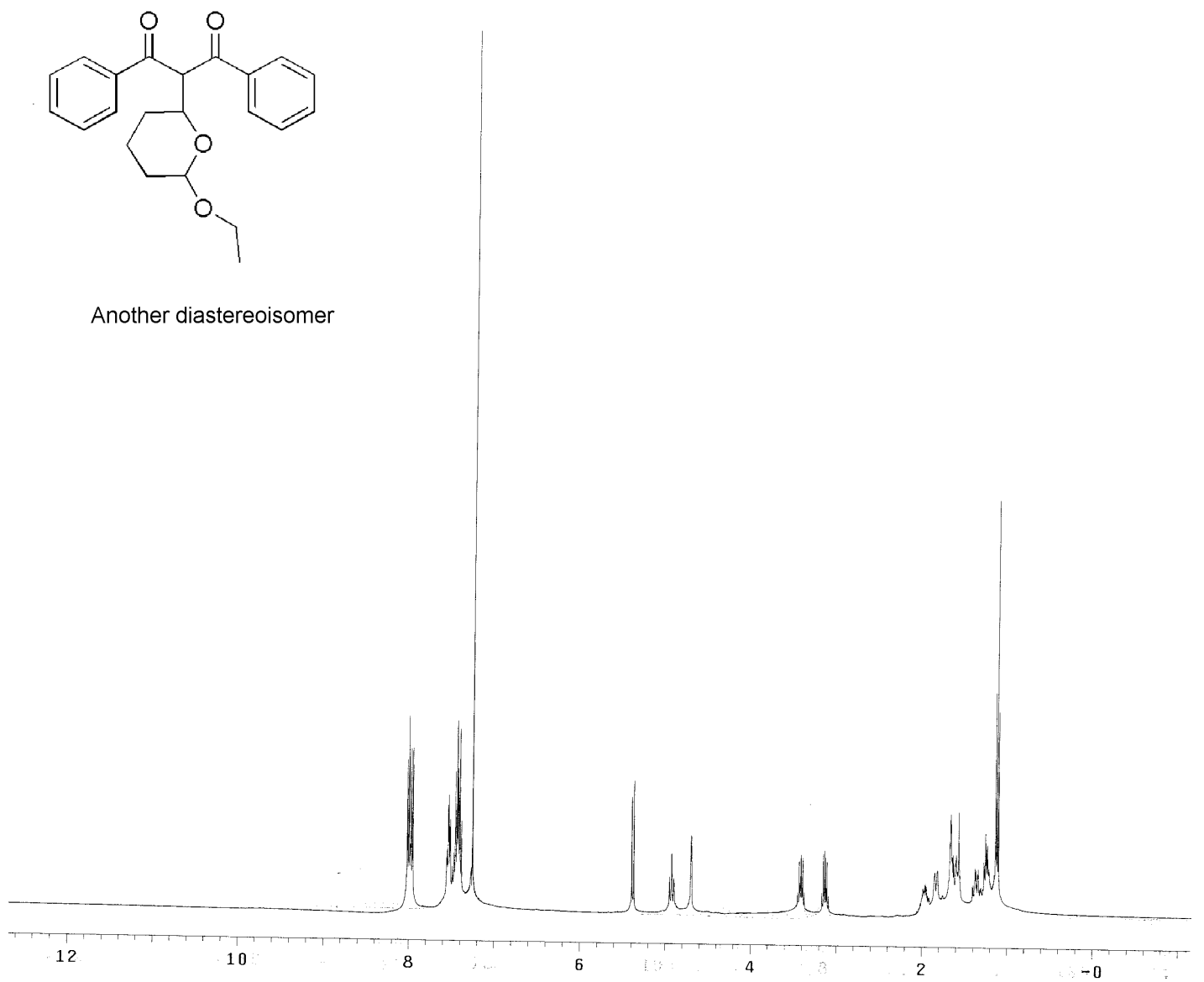




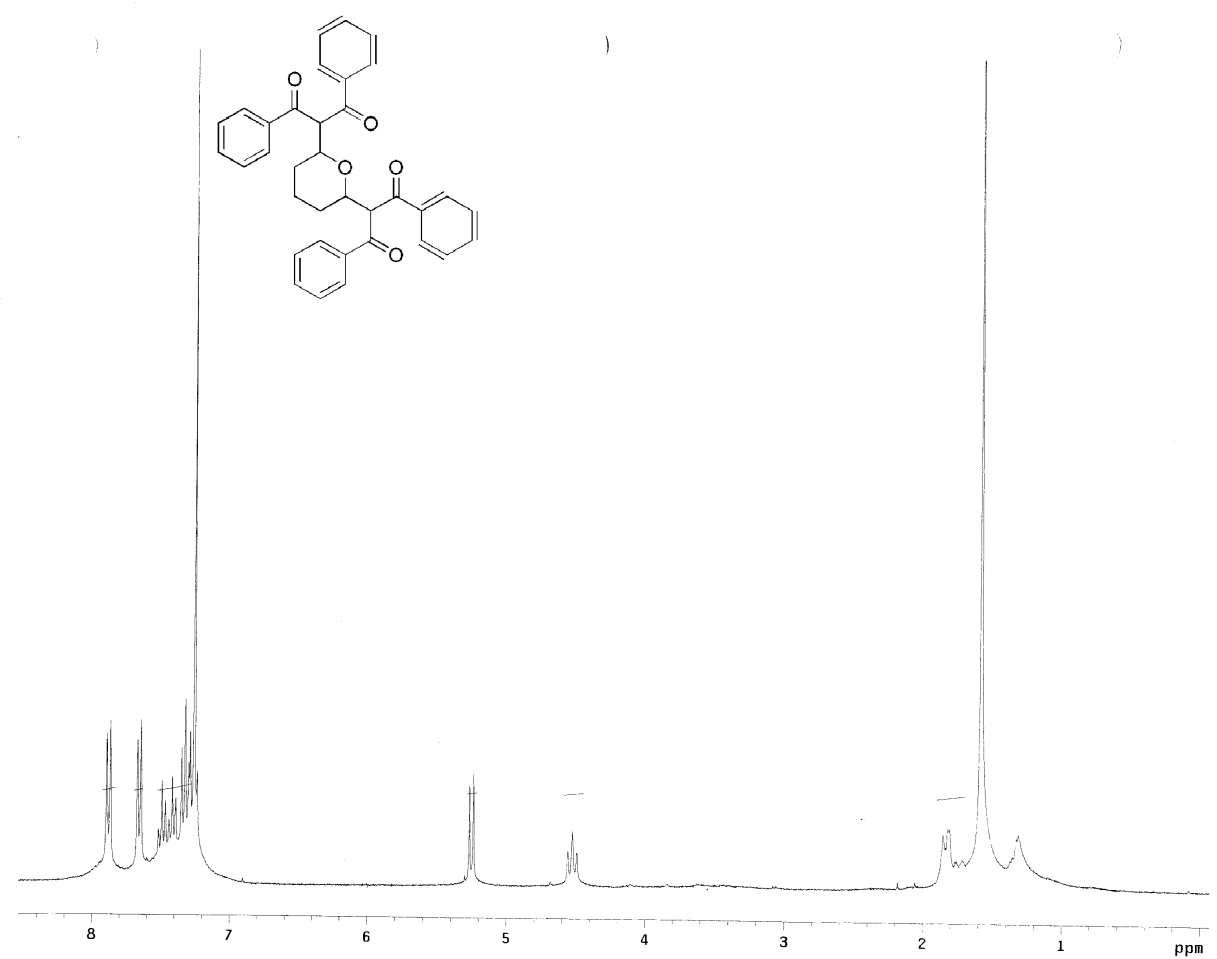



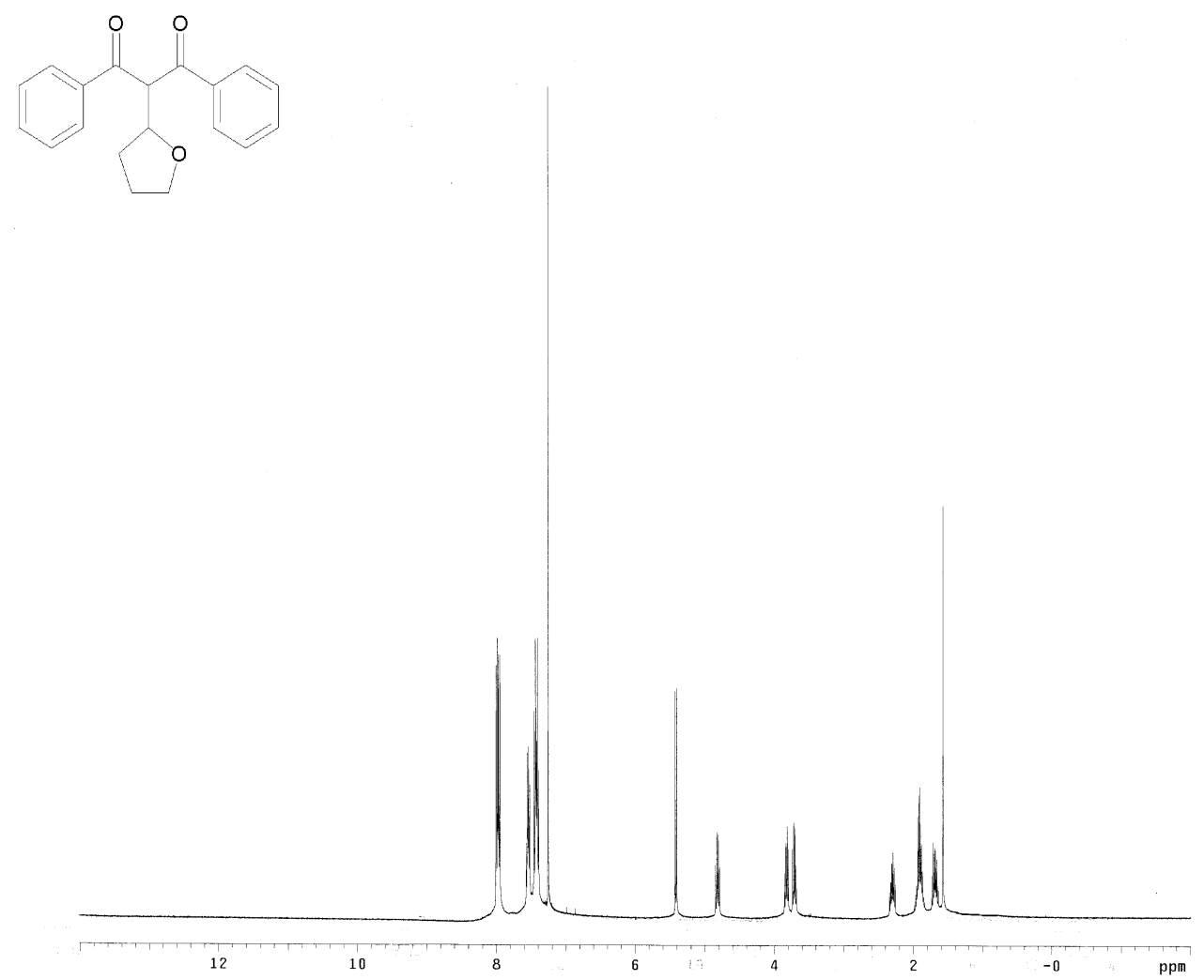


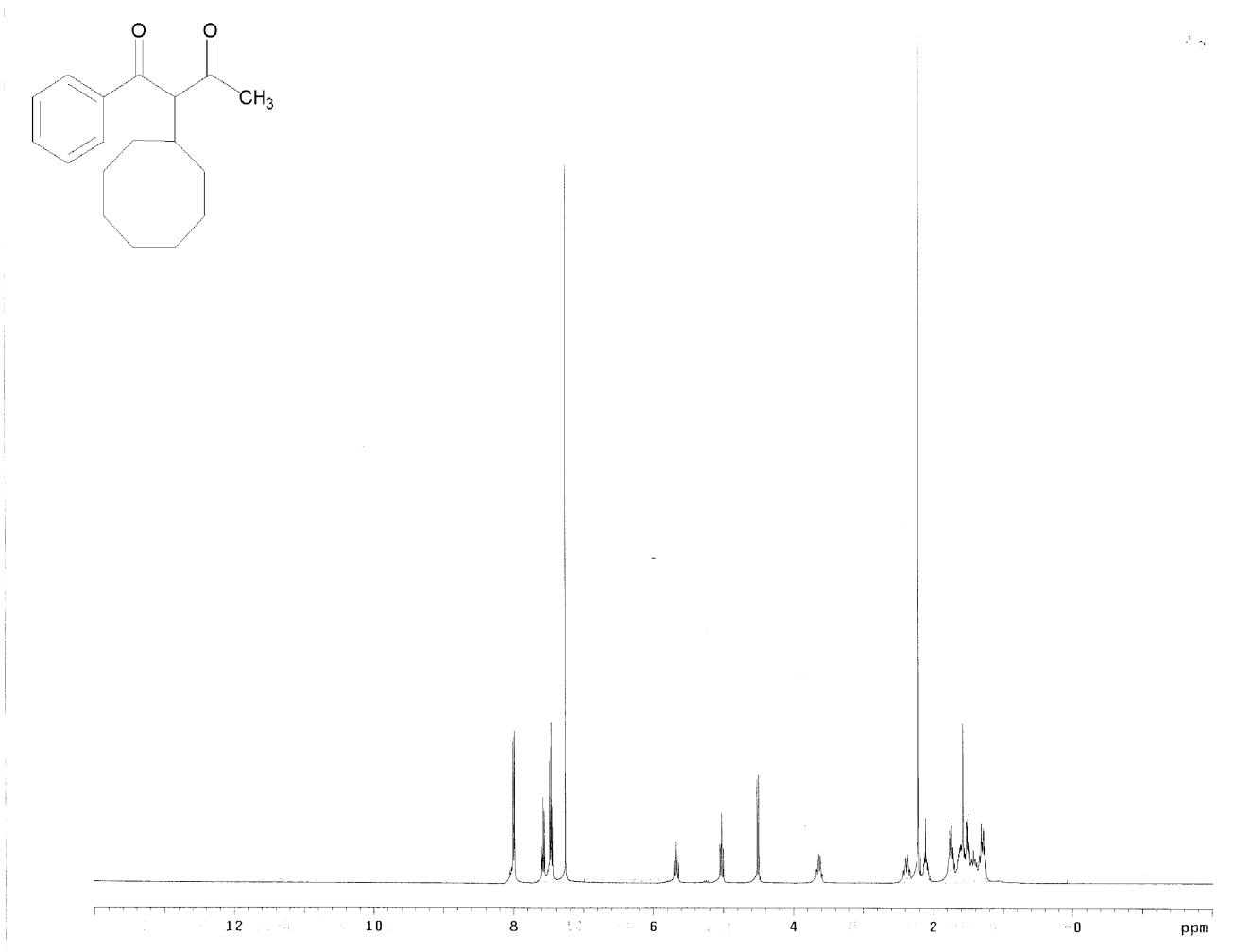



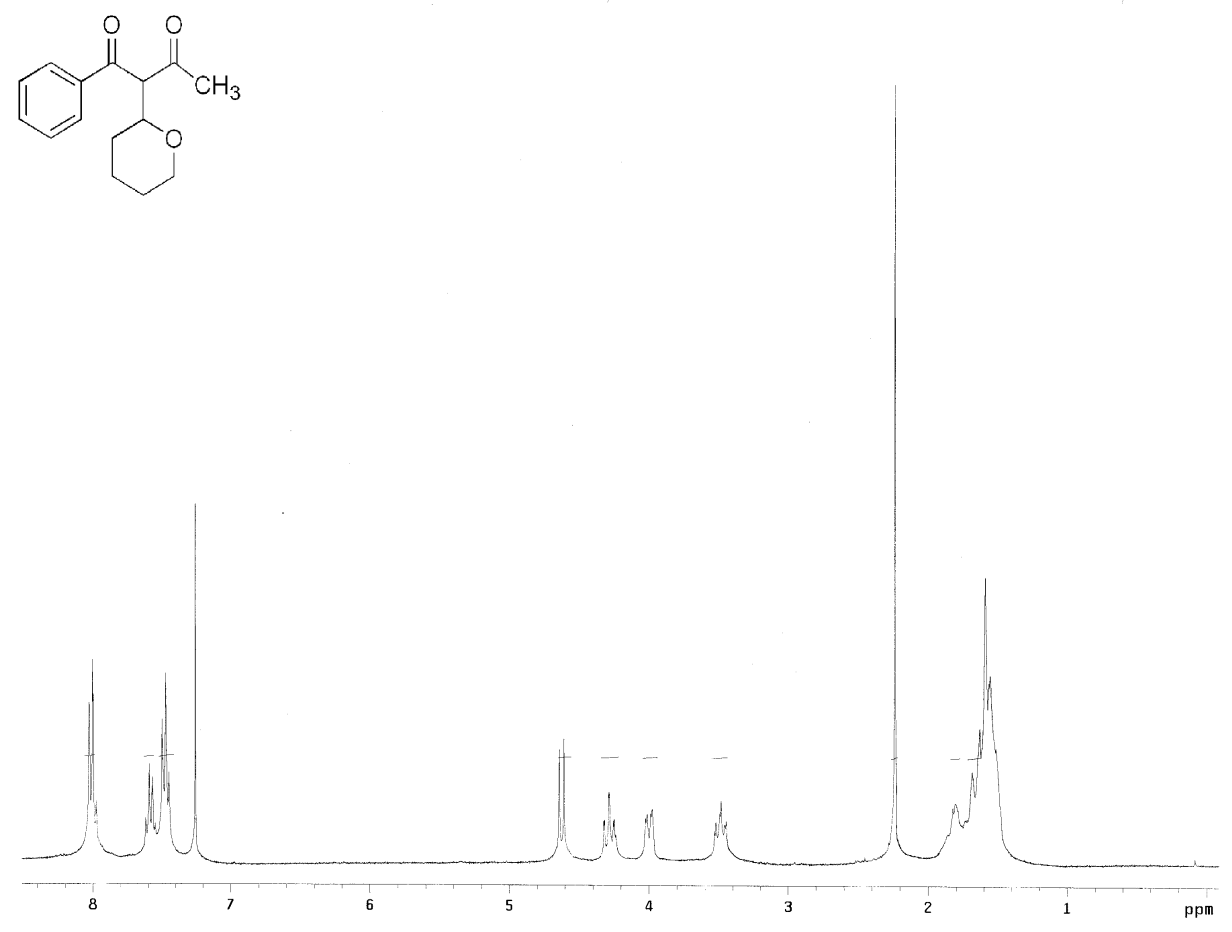

S23 